\title{
Successful treatment of refractory hyperferritinemic syndromes with canakinumab: a report of two cases
}

\author{
Riccardo Papa ${ }^{1,2^{*}} \mathbb{D}$, Valentina Natoli ${ }^{1}$, Roberta Caorsi ${ }^{3}$, Francesca Minoia ${ }^{4}$, Marco Gattorno ${ }^{3}$ and Angelo Ravelli ${ }^{1,3,5}$
}

\begin{abstract}
Background: Hyperferritinemic syndromes are systemic inflammatory disorders characterized by a dysfunctional immune response, which leads to excessive activation of the monocyte-macrophage system with hypercytokinemia and may pursue a rapidly fatal course.
\end{abstract}

Case presentation: We describe two patients of 11 and 9 years of age with hyperferritinemic syndromes, one with impending macrophage activation syndrome (MAS) and one with overt MAS, who were refractory or intolerant to conventional therapies, but improved dramatically with canakinumab.

Conclusions: Our report indicates that canakinumab may be efficacious in the management of hyperferritinemic syndromes, including MAS.

Keywords: Hyperferritinemic syndrome, Macrophage activation syndrome, Hemophagocytic lymphohistiocytosis, Hyperferritinemia, Anakinra, Canakinumab, Interleukin-1 inhibitors

\section{Background}

Hyperferritinemic syndromes (HFS) are systemic inflammatory disorders characterized by a dysfunctional immune response, leading to excessive activation of the monocyte-macrophage system with hypercytokinemia, and pronounced hemophagocytosis [1]. Serum ferritin level higher than $500 \mathrm{ng} / \mathrm{ml}$ is the common laboratory feature of this heterogeneous group of disorders, which ranges from rheumatic to non-rheumatic diseases, including primary immunodeficiencies, chronic infections, and malignancies.

The main condition within this disease spectrum is hemophagocytic lymphohistiocytosis (HLH), which is sub-classified into primary (or familial) HLH and secondary (or acquired or reactive) HLH [2]. The HLH

\footnotetext{
* Correspondence: riccardopapa@gaslini.org

'Università degli Studi di Genova, Genoa, Italy

${ }^{2}$ IRCCS Istituto Giannina Gaslini, Genoa, Italy

Full list of author information is available at the end of the article
}

associated with rheumatic illnesses is termed macrophage activation syndrome (MAS) [3, 4]. Among pediatric rheumatic diseases, MAS is encountered most commonly in children with systemic juvenile idiopathic arthritis (sJIA) [5]. Although many patients with active sJIA without MAS have ferritin levels exceeding 1000 $\mathrm{ng} / \mathrm{ml}[6]$, when MAS develops ferritin usually increases sharply. Beside the known conditions associated with HFS, in several instances no evident cause or underlying disease is observed. Timely recognition of HFS and prompt institution of an appropriate therapy are fundamental to avoid progression toward overt MAS.

In the past two decades, several well established criteria that can help to identify MAS in its early stage have been published [7-10]. Conversely, the management of the syndrome is not standardized and no universally agreed therapeutic protocols exist. Although high-dose glucocorticoids and cyclosporine A (CSA) still represent the mainstay of the treatment, instances of MAS that are refractory to these therapies are often encountered. 
Recently, a number of cases of sJIA-associated MAS dramatically benefiting from the interleukin (IL)-1 receptor antagonist anakinra (ANK) after inadequate response to glucocorticoids and CSA have been reported [11-17]. However, most patients needed dose escalation, up to $10 \mathrm{mg} / \mathrm{kg} / \mathrm{day}$, to control symptoms [18]. Based on these data, there is now wide agreement that ANK is a valuable medication for MAS.

The role of the IL-1 $\beta$ antibody canakinumab (CNK) is less clear, due to both the lack of experience with its use as interventional therapy in MAS and the occurrence of instances of MAS, recorded as adverse event, in the randomized clinical trials that led to its registration in sJIA $[19,20]$. However, the incidence of MAS in the trials was similar to the incidence of MAS in sJIA patients reported from a tertiary care pediatric rheumatology center in the US, which suggested that IL-1 inhibition with $\mathrm{CNK}$ does not have a major effect on the risk of developing MAS. In addition, many of the instances of MAS appeared to be triggered by an infection [21]. Recently, three patients with sJIA associated MAS which was refractory to conventional therapies or could not be controlled with standard doses of ANK or CNK, but responded dramatically to one single injection of $\mathrm{CNK}$ at higher doses $(7,5$ to $15 \mathrm{mg} / \mathrm{kg})$ have been reported in a meeting abstract [22].

In the present paper, we describe two patients with HFS, one with sJIA-like illness and impending MAS and one with sJIA and overt MAS, who were resistant or intolerant to conventional therapies, but improved rapidly with the administration of CNK.

\section{Case presentation}

\section{Patient 1}

A previously healthy 11-year-old boy was admitted to his local hospital with a 1-week history of fever (maximum temperature $39.4{ }^{\circ} \mathrm{C}$ ), urticarial rash and arthralgia, which did not improve with nonsteroidal anti-inflammatory, antihistamine and antibiotic therapy. On physical examination, he had generalized lymphadenopathy, but no evidence of overt arthritis. Body temperature was $38.7^{\circ} \mathrm{C}$. Laboratory tests showed increased acute phase reactants, anemia, and marked hyperferritinemia. Liver and kidney function tests, triglycerides, serum complement fractions, rheumatoid factor and antinuclear antibodies were all normal or negative. Abdominal ultrasound revealed diffuse lymph nodes enlargement and positron-emission tomography increased concentration of the radioactive tracer in the supraclavicular, mediastinal and abdominal lymph nodes. Chest radiograph, echocardiography, extensive infectious serology and autoantibodies were negative. Bone marrow aspirate disclosed expansion of the myeloid cell line and cervical lymph node biopsy a nonspecific reactive $\mathrm{B}$ and $\mathrm{T}$ lymphocyte hyperplasia. Next generation sequencing panel for monogenic autoinflammatory diseases and primary HLH as well as interferon signature were all negative. On the fifth day of admission, glucocorticoid therapy with $2 \mathrm{mg} / \mathrm{kg} /$ day prednisone was started, which led to defervescence of fever in 3 days and rapid improvement in clinical and laboratory abnormalities.

Six months later, after the decrease of prednisone dose to $0.15 \mathrm{mg} / \mathrm{kg} /$ day, there was recurrence of urticarial rash and diffuse arthralgia, without fever. The boy was then admitted to our hospital. Laboratory tests disclosed leukocytosis with neutrophilia, anemia, thrombocytosis, and increased erythrocyte sedimentation rate, $\mathrm{C}$-reactive protein (CRP), aspartate aminotransferase (AST), lactate dehydrogenase and ferritin (Table 1, T0). Because the illness did not meet both the International League for Associations of Rheumatology (ILAR) criteria for sJIA [23], due of the absence of arthritis and fever, and the 2016 classification criteria for MAS complicating sJIA [9], due to the lack of fever, a diagnosis of systemic juvenile idiopathic arthritis (JIA)-like hyperferritinemic syndrome was made. Treatment with intravenous methylprednisolone at $3 \mathrm{mg} / \mathrm{kg} /$ day administered in three divided doses and ANK at $5 \mathrm{mg} / \mathrm{kg} /$ day (i.e. 100 mg twice a day) was initiated. However, ANK had to be first tapered to $100 \mathrm{mg} /$ day and then discontinued due to the development of severe, intractable injection site reactions during the first week of treatment. In substitution of ANK, oral CSA at $4 \mathrm{mg} / \mathrm{kg} /$ day in two daily doses was started on the 7 th day of hospitalization. Over the subsequent week, there was improvement in laboratory changes (Table 1, T+14) and methylprednisolone dose was, then, gradually diminished.

However, on the 45th day of continuous hospitalization, although the boy had remained afebrile, there was worsening of his clinical conditions, with malaise, fatigue and diffuse arthralgia, and laboratory tests showed sharp increase in white blood cells, neutrophil count and ferritin, together with decreased hemoglobin and elevated AST (Table 1, T+45). At this stage, the 2016 classification criteria for MAS were still not fulfilled, but the child was thought to have impending MAS. We, then, decided to increase methylprednisolone dose to $3 \mathrm{mg} / \mathrm{kg} /$ day and to start CNK at $4 \mathrm{mg} / \mathrm{kg}$ subcutaneously, while continuing CSA. After the first injection of CNK, there was quick and striking improvement of laboratory abnormalities, with decrease in ferritin value from 18,000 to $4099 \mathrm{ng} / \mathrm{ml}$ and normalization of white blood cell and neutrophil count within 5 days (Table 1, T+50). One month later, the blood cell counts, the acute phase reactants and the AST had returned to normal and the level of ferritin was 
Table 1 Course of laboratory tests over time in patient 1

\begin{tabular}{|c|c|c|c|c|c|}
\hline Days from admission at Authors' center & 0 & +14 & $+45^{\mathrm{a}}$ & +50 & +80 \\
\hline White blood cells $\left(\times 10^{3} / \mathrm{mm}^{3}\right)$ & 17.9 & 10.4 & 44.5 & 11.4 & 8.9 \\
\hline Neutrophil count $\left(\times 10^{3} / \mathrm{mm}^{3}\right)$ & 15.0 & 8.9 & 41.7 & 6.4 & 5.0 \\
\hline Hemoglobin (g/dL) & 10.3 & 10.6 & 9.3 & 9.8 & 12.5 \\
\hline Platelet count $\left(\times 10^{3} / \mathrm{mm}^{3}\right)$ & 482 & 320 & 418 & 459 & 315 \\
\hline Erythrocyte sedimentation rate $(\mathrm{mm} / \mathrm{h})$ & 59 & 37 & 57 & 61 & 18 \\
\hline C-reactive protein (mg/dL) & 8.2 & 4.9 & 11.5 & 1.5 & $<0.46$ \\
\hline Aspartate aminotransferase $(\mathrm{U} / \mathrm{L})$ & 229 & 68 & 170 & 127 & 12 \\
\hline Lactate dehydrogenase (U/L) & 1752 & 951 & 956 & 862 & 853 \\
\hline Triglycerides (mg/dL) & 112 & 158 & 133 & 226 & 107 \\
\hline Fibrinogen (mg/dL) & 341 & 442 & 415 & 251 & 287 \\
\hline Ferritin $(\mathrm{ng} / \mathrm{mL})$ & 13,200 & 8550 & 18,000 & 4099 & 581 \\
\hline
\end{tabular}

${ }^{\mathrm{a}}$ Start of canakinumab administration

markedly reduced (Table $1, \mathrm{~T}+80$ ). The boy was, then, discharged with monthly $\mathrm{CNK}$ administration, in association with CSA and tapering oral prednisone.

\section{Patient 2}

A 9-year-old girl was diagnosed with sJIA and MAS at her local hospital based on the presence of high-spiking fever, erythematous rash and polyarthritis, together with increased acute phase reactants, AST, triglycerides and ferritin, and decreased platelet count and fibrinogen. She was given high-dose intravenous methylprednisolone at $30 \mathrm{mg} / \mathrm{kg}$ for 3 consecutive days, which led to improvement in clinical manifestations and laboratory abnormalities. However, shortly after glucocorticoid therapy had been switched to oral prednisone there was a recurrence of MAS features and the girl was transferred to the regional tertiary care hospital.

On admission, a full-blown clinical and laboratory picture of MAS was detected. Three additional pulses of intravenous methylprednisolone at $30 \mathrm{mg} / \mathrm{kg}$ were, then, administered and CSA at $4 \mathrm{mg} / \mathrm{kg} /$ day, with $\mathrm{ANK}$ at 6 $\mathrm{mg} / \mathrm{kg} /$ day, were started. This treatment led to rapid improvement in MAS features. However, during hospitalization the girl developed a thrombophlebitis in the right arm in the site of a venipuncture and developed fever, malaise and chest pain in spite of antibiotic therapy. A few days later, a chest radiograph disclosed marked cardiomegaly, which was found on echocardiography to be due to massive pericardial effusion. This complication was followed by recurrence of MAS abnormalities, with increase in ferritin to $20,350 \mathrm{ng} / \mathrm{ml}$ (Table 2, $\mathrm{T}-4$ ). The girl was given 3 additional pulses of intravenous methylprednisolone at $30 \mathrm{mg} / \mathrm{kg}$ and was, then, transferred to our hospital for further care.

On admission, the girl was afebrile and laboratory tests revealed an overall improvement of MAS abnormalities as compared with previous assessment (Table 2, T0). Treatment was, then, continued with intravenous methylprednisolone at $2 \mathrm{mg} / \mathrm{kg} /$ day, together with CSA and ANK at unchanged doses. In the meantime, pericardial

Table 2 Course of laboratory tests over time in patient 2

\begin{tabular}{|c|c|c|c|c|c|c|c|}
\hline Days from admission at Authors' center & -4 & 0 & +20 & +30 & $+35^{a}$ & +40 & +50 \\
\hline White blood cells $\left(\times 10^{3} / \mathrm{mm}^{3}\right)$ & 14.2 & 10.6 & 3.4 & 5.4 & 3.7 & 18.9 & 5.5 \\
\hline Neutrophil count $\left(\times 10^{3} / \mathrm{mm}^{3}\right)$ & 11.9 & 6.6 & 1.9 & 3.4 & 1.7 & 15.1 & 2.9 \\
\hline Hemoglobin (g/dL) & 9.2 & 11.2 & 10.8 & 10.8 & 10.5 & 9.9 & 11.1 \\
\hline Platelet count $\left(\times 10^{3} / \mathrm{mm}^{3}\right)$ & 285 & 424 & 105 & 233 & 145 & 164 & 253 \\
\hline Erythrocyte sedimentation rate (mm/h) & 75 & 68 & 43 & 55 & 63 & 50 & 36 \\
\hline C-reactive protein (mg/dL) & 2.5 & $<0.46$ & 1.83 & $<0.46$ & 0.6 & $<0.46$ & $<0.46$ \\
\hline Aspartate aminotransferase $(U / L)$ & 61 & 40 & 350 & 111 & 113 & 35 & 31 \\
\hline Lactate dehydrogenase (U/L) & 1673 & 1048 & 4960 & 1541 & 2122 & 1116 & 468 \\
\hline Triglycerides (mg/dL) & 209 & 173 & 273 & - & 157 & - & - \\
\hline Fibrinogen (mg/dL) & 356 & 418 & 234 & 297 & 424 & 312 & 267 \\
\hline Ferritin (ng/mL) & 20,350 & 5065 & 49,700 & 17,580 & 28,634 & 12,340 & 201 \\
\hline
\end{tabular}

${ }^{\mathrm{a}}$ Start of canakinumab administration 
drainage revealed the purulent nature of the accumulated fluid, whose culture led to the isolation of a methicillin-resistant Staphylococcus aureus. Therapy with large-spectrum antibiotics was started. However, on the third week of hospitalization there was a sudden worsening of clinical conditions, with recurrence of fever and malaise. Repeated laboratory tests showed a fullblown picture of MAS, with sharply elevated ferritin (Table 2, T +20 ), which met the 2016 classification criteria for the syndrome. Treatment consisted of 3 consecutive days of methylprednisolone at $30 \mathrm{mg} / \mathrm{kg}$ plus intravenous immunoglobulin at $2 \mathrm{~g} / \mathrm{kg}$. Cyclosporine A and ANK were continued, but the dose of the latter medication was raised to $8 \mathrm{mg} / \mathrm{kg} /$ day. The laboratory picture of MAS improved (Table 2, $\mathrm{T}+30$ ), but after the shift of intravenous methylprednisolone to the conventional regimen of $2 \mathrm{mg} / \mathrm{kg} /$ day and surgical pericardiotomy, which led to the evacuation of $350 \mathrm{ml}$ of purulent fluid, there was another flare of MAS, with a further increase in ferritin to $28,634 \mathrm{ng} / \mathrm{ml}$ (Table 2, $\mathrm{T}+35$ ). Due to the presence of major signs of corticosteroid toxicity and the inefficacy of ongoing treatment in preventing MAS recurrences, we decided to start CNK at $5 \mathrm{mg} / \mathrm{kg}$. Anakinra was stopped and shortly afterward also CSA was discontinued. The first injection of $\mathrm{CNK}$ was followed by prompt improvement in all MAS biomarkers and the girl could be discharged 2 weeks later in good general condition, with normal ferritin level (Table 2, $\mathrm{T}+50)$ and on tapering oral prednisone. Nine months later she was receiving low-dose prednisone and monthly CNK and had experienced no relapses of either SIIA or MAS.

\section{Discussion and conclusions}

We have described two patients with HFS, which were refractory or intolerant to conventional therapies, but responded dramatically to the administration of CNK. The first patient had a long-standing inflammatory illness closely resembling sJIA, but without overt arthritis. Although the 2016 classification criteria for MAS [9] were not met, he had a sharp increase in ferritin level, which was thought to herald the potential occurrence of full-blown MAS. Although initial ANK therapy, in conjunction with intravenous methylprednisolone and CSA, was followed by improvement in laboratory abnormalities, especially hyperferritinemia, the subsequent discontinuation of ANK because of serious and intractable injection site reaction was followed by worsening of the condition, which was reversed quickly after a single injection of CNK. The second patient had recurrent episodes of MAS, the last of which was precipitated after surgical pericardiotomy to evacuate purulent pericardial fluid and was not controlled by the combination of highdose intravenous methylprednisolone, CSA and ANK.
The administration of CNK led to rapid recovery of all clinical and laboratory features of MAS.

The rationale for the use of cytokine blockers in the management of MAS was provided by the demonstration of the prominent role of the proinflammatory cytokines targeted by these agents, namely TNF- $\alpha$, IL-1 and IL-6, in the pathophysiology of the syndrome [4, 18]. The first cytokine inhibitors used to treat MAS were TNF blockers. However, after initial encouraging reports, subsequent experiences indicated that these agents may trigger MAS in some instances. Nowadays, TNF inhibition is not considered the ideal therapy for MAS, whereas there is much more interest for therapy directed at two other proinflammatory cytokines, IL-1 and IL-6 [18].

Thus far, the information on the use of IL- 6 blockade in the management of MAS is still limited, given the reports from the tocilizumab clinical trials and post-marketing surveillance, which suggest that IL-6 inhibition does not provide full protection against MAS [21]. By contrast, substantial benefit from ANK treatment in sJIA-associated MAS after inadequate response to glucocorticoids and CSA has been described in many case reports [24, 25]. Notably, occurrence of MAS was occasionally seen in children treated with doses of $1-2 \mathrm{mg} / \mathrm{kg}$ daily [26, 27]. In some of these patients, however, features of MAS improved after the dose of ANK was escalated. There is now widespread consensus that ANK, particularly at higher doses, might be effective at least in some patients with sJIA-associated MAS [18].

Our report suggests that the monoclonal IL-1 $\beta$ antibody CNK may represent an alternative therapeutic option for children with MAS who are refractory or intolerant to conventional therapies and for select critically ill children with HFS who do not meet neither sJIA nor MAS criteria. The efficacy of CNK in ANK-resistant patients with HFS has been previously reported [28]. The better effectiveness of CNK may be due to differences in molecular targets (i.e. ANK blocks both IL- $1 \alpha$ and IL-1 $\beta$ ) or pharmacokinetics (i.e. longer half-life of $\mathrm{CNK}$ ). Further clinical experience is needed to confirm our observations and to clarify whether a dose of CNK higher than the $4 \mathrm{mg} /$ $\mathrm{kg}$ used in the routine management of sJIA is needed to control HFS, including MAS.

\section{Abbreviations}

HFS : Hyperferrritinemic syndromes; HLH : Hemophagocytic lymphohistiocytosis; MAS : Macrophage activation syndrome; sJIA : Systemic juvenile idiopathic arthritis; ANK : Anakinra; CSA : Cyclosporine A; CNK : Canakinumab; IL : Interleukin

Acknowledgements

Not applicable. 


\section{Authors' contributions}

$R P, V N$ and $A R$ analyzed and interpreted the patient data and drafted the manuscript. RC, FM and MG examined the patients and reviewed drafts. All authors read and approved the final manuscript.

\section{Funding}

The Authors declare that no funding was available for this manuscript.

\section{Availability of data and materials}

The datasets used and/or analyzed during the current study are available from the corresponding author on reasonable request.

\section{Ethics approval and consent to participate}

Not applicable.

\section{Consent for publication}

Informed consent was obtained from the patient's parents for publication of this case report.

\section{Competing interests}

MG has received grant support and/or speaking or consultant fees from SOBI and Novartis. AR has received grant support and/or speaking or consultant fees from AbbVie, Bristol-Myers Squibb, Novartis, Pfizer, Roche and Johnson \& Johnson. The other authors do not have conflict of interests to declare.

\section{Author details}

${ }^{1}$ Università degli Studi di Genova, Genoa, Italy. ${ }^{2}$ IRCCS Istituto Giannina Gaslini, Genoa, Italy. ${ }^{3}$ None, Italy. ${ }^{4}$ Fondazione IRCCS Ca' Granda Ospedale Maggiore Policlinico, Milan, Italy. ${ }^{5}$ Sechenov First Moscow State Medical University, Moscow, Russian Federation.

Received: 12 February 2020 Accepted: 3 July 2020

Published online: 11 July 2020

\section{References}

1. Schulert GS, Canna SW. Convergent pathways of the hyperferritinemic syndromes. Int Immunol. 2018;30:195-203.

2. Al-Samkari H, Berliner N. Hemophagocytic Lymphohistiocytosis. Annu Rev Pathol. 2018;13:27-49.

3. Ravelli A, Davì S, Minoia F, Martini A, Cron RQ. Macrophage activation syndrome. Hematol Oncol Clin North Am. 2015;29:927-41.

4. Crayne CB, Albeituni S, Nichols KE, Cron RQ. The immunology of macrophage activation syndrome. Front Immunol. 2019;10:119.

5. Lee JJY, Schneider R. Systemic juvenile idiopathic arthritis. Pediatr Clin N Am. 2018;65:691-709.

6. Nirmala N, Grom A, Gram H. Biomarkers in systemic juvenile idiopathic arthritis: a comparison with biomarkers in Cryopyrin associated periodic syndromes. Curr Opin Rheumatol. 2014;26:545-52.

7. Henter J-I, Horne A, Aricó M, Egeler M, Filipovich AH, Imashuku S, et al. HLH2004: diagnostic and therapeutic guidelines for hemophagocytic lymphohistiocytosis. Pediatr Blood Cancer. 2007;48:124-31.

8. Ravelli A, Magni-Manzoni S, Pistorio A, Besana C, Foti T, Ruperto N, et al. Preliminary diagnostic guidelines for macrophage activation syndrome complicating systemic juvenile idiopathic arthritis. J Pediatr. 2005:146:598-604.

9. Ravelli A, Minoia F, Davì S, Horne AC, Bovis F, Pistorio A, et al. Classification criteria for macrophage activation syndrome complicating systemic juvenile idiopathic arthritis: a European league against rheumatism/American College of Rheumatology/Paediatric rheumatology international trials organisation collaboration. Arthritis Rheumatol. 2016;68:566-76.

10. Minoia F, Bovis F, Davì S, Horne AC, Fischbach M, Frosch M, et al. Development and initial validation of the MS score for diagnosis of macrophage activation syndrome in systemic juvenile idiopathic arthritis. Ann Rheum Dis. 2019;78:1357-62

11. Nigrovic PA, Mannion M, Prince FHM, Zeft A, Rabinovich CE, van Rossum MAJ, et al. Anakinra as first-line disease-modifying therapy in systemic juvenile idiopathic arthritis: report of forty-six patients from an international multicenter series. Arthritis Rheum. 2011;63:545-55.

12. Miettunen PM, Narendran A, Jayanthan A, Behrens EM, Cron RQ. Successful treatment of severe paediatric rheumatic disease-associated macrophage activation syndrome with interleukin-1 inhibition following conventional immunosuppressive therapy: case series with 12 patients. Rheumatology (Oxford). 2011:50:417-9.

13. Kelly A, Ramanan AV. A case of macrophage activation syndrome successfully treated with anakinra. Nat Clin Pract Rheumatol. 2008:4:615-20.

14. Durand M, Troyanov $Y$, Laflamme P, Gregoire G. Macrophage activation syndrome treated with anakinra. J Rheumatol. 2010;37:879-80.

15. Bruck N, Suttorp M, Kabus M, Heubner G, Gahr M, Pessler F. Rapid and sustained remission of systemic juvenile idiopathic arthritis-associated macrophage activation syndrome through treatment with anakinra and corticosteroids. J Clin Rheumatol Pract Rep Rheum Musculoskelet Dis. 2011; 17:23-7.

16. Kahn PJ, Cron RQ. Higher-dose Anakinra is effective in a case of medically refractory macrophage activation syndrome. J Rheumatol. 2013;40:743-4.

17. Record $J$, Beukelman T, Cron RQ. Combination therapy of abatacept and anakinra in children with refractory systemic juvenile idiopathic arthritis: a retrospective case series. J Rheumatol. 2011;38:180-1.

18. Ravelli A, Grom AA, Behrens EM, Cron RQ. Macrophage activation syndrome as part of systemic juvenile idiopathic arthritis: diagnosis, genetics, pathophysiology and treatment. Genes Immun. 2012;13:289-98.

19. Ruperto N, Brunner HI, Quartier P, Constantin T, Wulffraat N, Horneff G, et al. Two randomized trials of Canakinumab in systemic juvenile idiopathic arthritis. N Engl J Med. 2012;367:2396-406.

20. Grom AA, llowite NT, Pascual V, Brunner HI, Martini A, Lovell D, et al. Rate and clinical presentation of macrophage activation syndrome in patients with systemic juvenile idiopathic arthritis treated with Canakinumab. Arthritis Rheumatol Hoboken NJ. 2016:68:218-28.

21. Grom AA, Horne A, De Benedetti F. Macrophage activation syndrome in the era of biologic therapy. Nat Rev Rheumatol. 2016;12:259-68.

22. Kostik M, Snegireva L, Dubko M, Masalova V, Likhacheva T, Kornishina T, et al. A98: rescue treatment by increased doses of IL-1 inhibitors for macrophage activation syndrome in children with systemic juvenile idiopathic arthritis [abstract]. Arthritis Rheumatol. 2014;66:S133.

23. Petty RE, Southwood TR, Manners P, Baum J, Glass DN, Goldenberg J, et al. International league of associations for rheumatology classification of juvenile idiopathic arthritis: second revision, Edmonton, 2001. J Rheumatol. 2004;31:390-2.

24. Möller JC, Paul D, Ganser G, Range U, Gahr M, Kelsch R, et al. IL10 promoter polymorphisms are associated with systemic onset juvenile idiopathic arthritis (SoJIA). Clin Exp Rheumatol. 2010;28:912-8.

25. Krebs P, Crozat K, Popkin D, Oldstone MB, Beutler B. Disruption of MyD88 signaling suppresses hemophagocytic lymphohistiocytosis in mice. Blood. 2011;117:6582-8.

26. Hayashi K, Jin Z, Onoda S, Joko H, Teramoto N, Ohara N, et al. Rabbit model for human EBV-associated hemophagocytic syndrome (HPS): sequential autopsy analysis and characterization of IL-2-dependent cell lines established from herpesvirus papio-induced fatal rabbit lymphoproliferative diseases with HPS. Am J Pathol. 2003;162:1721-36.

27. Hsieh W-C, Chang Y, Hsu M-C, Lan B-S, Hsiao G-C, Chuang H-C, et al. Emergence of anti-red blood cell antibodies triggers red cell phagocytosis by activated macrophages in a rabbit model of Epstein-Barr virus-associated hemophagocytic syndrome. Am J Pathol. 2007;170:1629-39.

28. Tucker LB, Lamot L, Niemirtz I, Chung BK, Cabral DA, Houghton K, et al. Complexity in unclassified autoinflammatory disease: a case report illustrating the potential for disease arising from the allelic burden of multiple variants. Pediatr Rheumatol Online J. 2019;17:70-7.

\section{Publisher's Note}

Springer Nature remains neutral with regard to jurisdictional claims in published maps and institutional affiliations. 